\title{
STELLAR X-RAY SOURCES
}

\author{
HARVEY D. TANANBAUM* \\ American Science \& Engineering, Cambridge, Mass., U.S.A.
}

\begin{abstract}
Data are presented for Cygnus X-1, Cygnus X-3, and Scorpius X-1 from radio to X-ray wavelengths. The evidence for Cygnus $X-1$ 's being a black hole is now quite convincing. New data for Cygnus $\mathrm{X}-3$ show the presence of X-ray activity at the time of the giant radio outburst. The data for Scorpius X-1 show a close correlation between the $\mathrm{X}$-ray and optical behavior, but coverage was not sufficiently complete to fully assess the relationship (if any) between the X-ray and optical emission and the radio emission.
\end{abstract}

\section{Introduction}

At the IAU General Assembly the subject of binary X-ray sources was reviewed. The prevalence of intensity variations often on time scales as short as $0.1 \mathrm{~s}$ was established for the galactic X-ray sources, and the evidence for the binary nature of the galactic sources was presented.

For this meeting I have chosen for discussion three galactic X-ray sources: Cygnus $X-1$, Cygnus X-3 and Scorpius X-1. The reasons for choosing these sources were that all three have radio counterparts, all three $\mathrm{X}$-ray and radio sources have been fairly extensively studied, and the three objects have different appearances. Without attempting to link the behavior of these three sources, what I would like to do here is to look at them with the objective of assessing whether the radio emission and the X-ray emission are in any way related.

As I began to work on the paper, I approached it with the belief that the X-ray and radio emission were essentially unrelated. About the only relationship that had been observed was the appearance of a detectable radio flux that coincided within a week's time with a factor of 4 decrease in the $2-6 \mathrm{keV}$ emission from Cygnus X-1. As we shall see there is now some additional evidence suggesting that the radio emission is not totally decoupled from the $\mathrm{X}$-ray, although there are still very many unanswered questions.

\section{Cygnus X-1}

Perhaps the most significant of the Uhuru results for the galactic X-ray sources has been the discovery of pulsations from Cygnus X-1. These data led to further study of this object and to the present belief that we are dealing with a black hole. I would like to present the data we have on this object and consider the status of the black hole identification. Figure 1 contains data showing substantial variations in X-ray intensity on time scales from $100 \mathrm{~ms}$ to $10 \mathrm{~s}$. Some $80 \mathrm{~s}$ of data are shown here summed on four time scales from $100 \mathrm{~ms}$ up to $14 \mathrm{~s}$. I should point out that similar X-ray variability also reported by Rappaport et al. (1971a), Holt et al. (1971) and Shulman et al. (1971)

* Now at Center for Astrophysics, Harvard College Observatory/Smithsonian Astrophysical Observatory, Cambridge Massachusetts. 

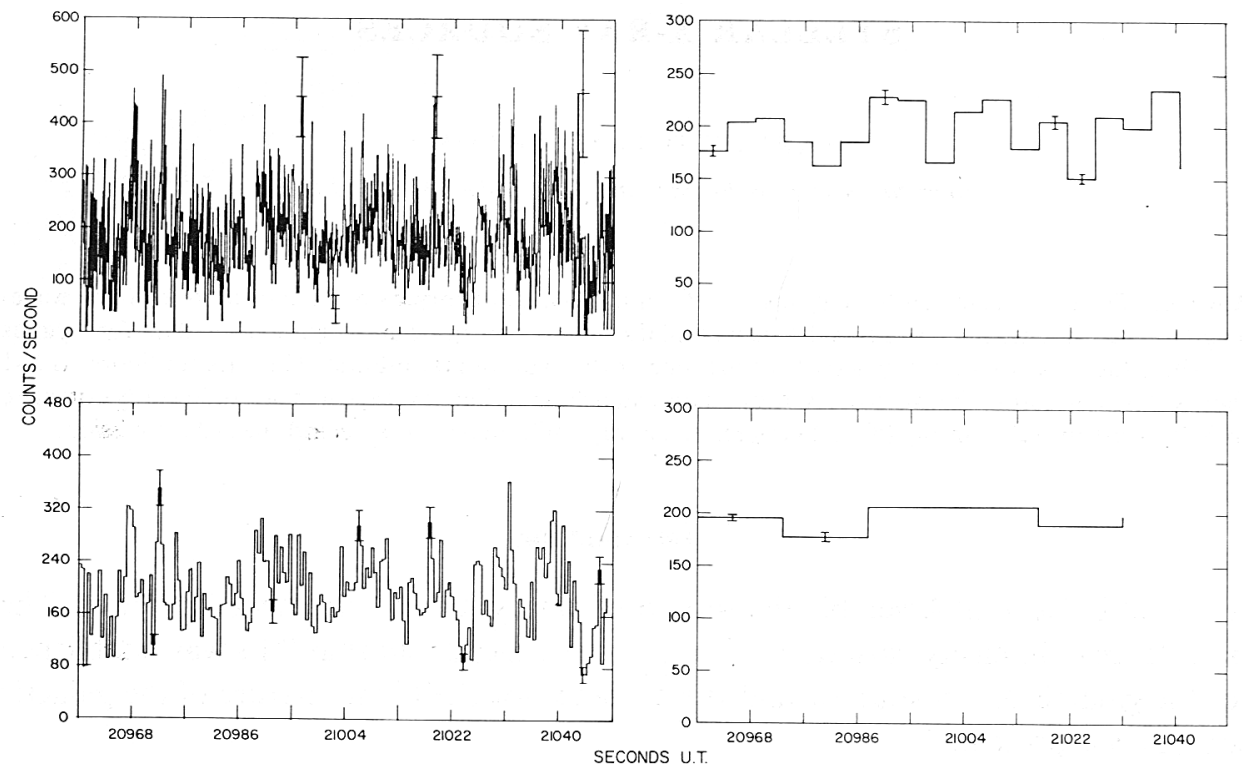

Fig. 1. Observations of Cygnus X-1 on 1971, June 10. Data have been corrected for the triangular collimator response. Data are summed over $0.096 \mathrm{~s}, 0.48-\mathrm{s}, 4.8-\mathrm{s}$, and 14.4-s intervals. Typical $1-\sigma$ error bars are shown.

compels us to consider a source region of $10^{9} \mathrm{~cm}$ or less. Figure 2 shows the X-ray location obtained from an MIT rocket flight (Rappaport et al., 1971b) and from Uhuru which led to the discovery of a radio source by Braes and Miley (1971) and by Hjellming and Wade (1971). It is this precise radio location that led to the optical identification by Webster and Murdin (1972) and by Bolton (1972a) of Cygnus X-1 with the 5.6-day spectroscopic binary system HDE 226868. The central object of this system is a 9th magnitude star, most likely a B0 supergiant, and first mass estimates for the primary led to a mass in excess of $3 M_{\odot}$ for the unseen companion. This came from the mass function determined from the absorption line velocities

$$
\frac{M_{\mathrm{s}}^{3} \sin ^{3} i}{\left(M_{\mathrm{s}}+M_{\mathrm{BO}}\right)^{2}} \simeq 0.2 \text {. }
$$

Even at $i=90^{\circ}$, which gives a minimum value of the mass of the secondary,

$$
M_{\mathrm{B} 0}=20 \rightarrow M_{\mathrm{s}}=5 M_{\odot}
$$

If the secondary is the compact X-ray source, then it could well be a black hole, a point to which we will return shortly.

We attempted to confirm the identification of the X-ray source with HDE 226868 by looking for a 5.6-day effect on the X-ray light curve. In December 1971 and January 1972 we observed Cygnus X-1 continuously for 35 days. We folded the X-ray data with many different periods including 5.6 days, and the results are shown in Figure 3. Data at 2-6 keV are shown folded modulo 3.0, 5.6, and 6.2 days; the average is indi- 


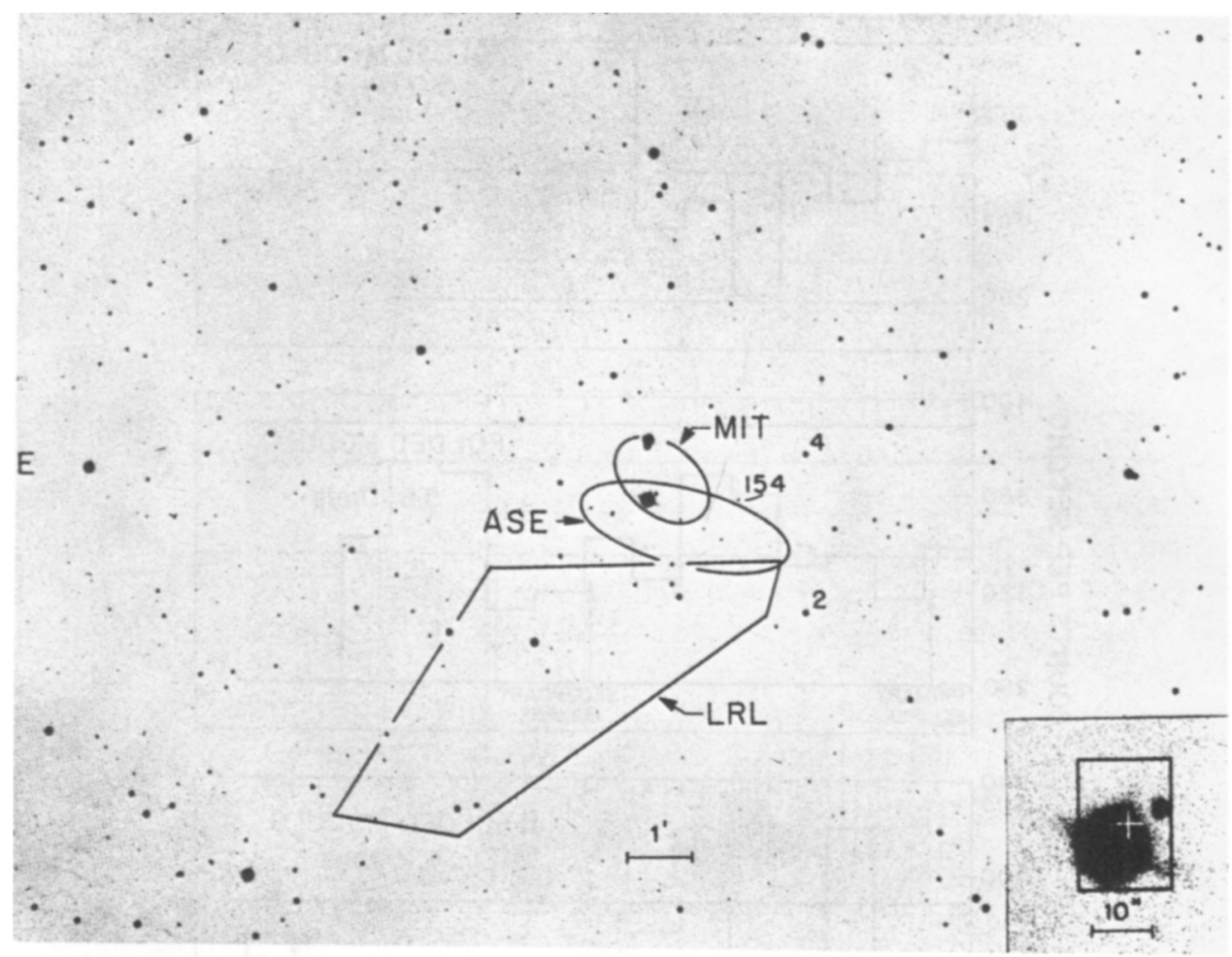

Fig. 2. X-ray location of Cygnus X-1. HDE 226868 is the bright star in the overlap between the MIT and ASE error boxes. The insert shows the radio location, which was reduced to an uncertainty of less than $1^{\prime \prime}$ after this figure was drawn and is coincident with HDE 226868.

cated by the dotted lines, and 2- $\sigma$ error bars are indicated by the solid lines. We conclude that there is no evidence for a 5.6-day eclipse here and believe that previous reports from balloon observations of such an effect at higher energies were caused by the large-scale time variability and not by a 5.6 day effect. The report by Professor Boyd at Sydney of a possible 5.6-day effect at energies below $3 \mathrm{keV}$ may be due to a real effect or may be due to the variability of the source and the presence of only two data points. In any case, the absence of a 5.6-day effect does not rule out the identification and can be understood in terms of an appropriate inclination angle for the orbital plane of the binary system.

With the use of Uhuru as an observatory, we have analyzed 16 months of data on Cygnus X-1, which are shown in Figure 4. We have plotted the $2-6 \mathrm{keV}$ intensity versus day of 1970 . The vertical lines for a given day are not error bars, but rather show the range of variability observed on that day. For some days we have only the average intensity shown by a dash available in our analyzed results. We see that a remarkable transition occurred in March and April 1971, with the source changing its average $2-6 \mathrm{keV}$ intensity level by a factor of 4 . We have also indicated in the figure the 6-10 keV and 10-20 keV X-ray intensities and see that the average level of the 

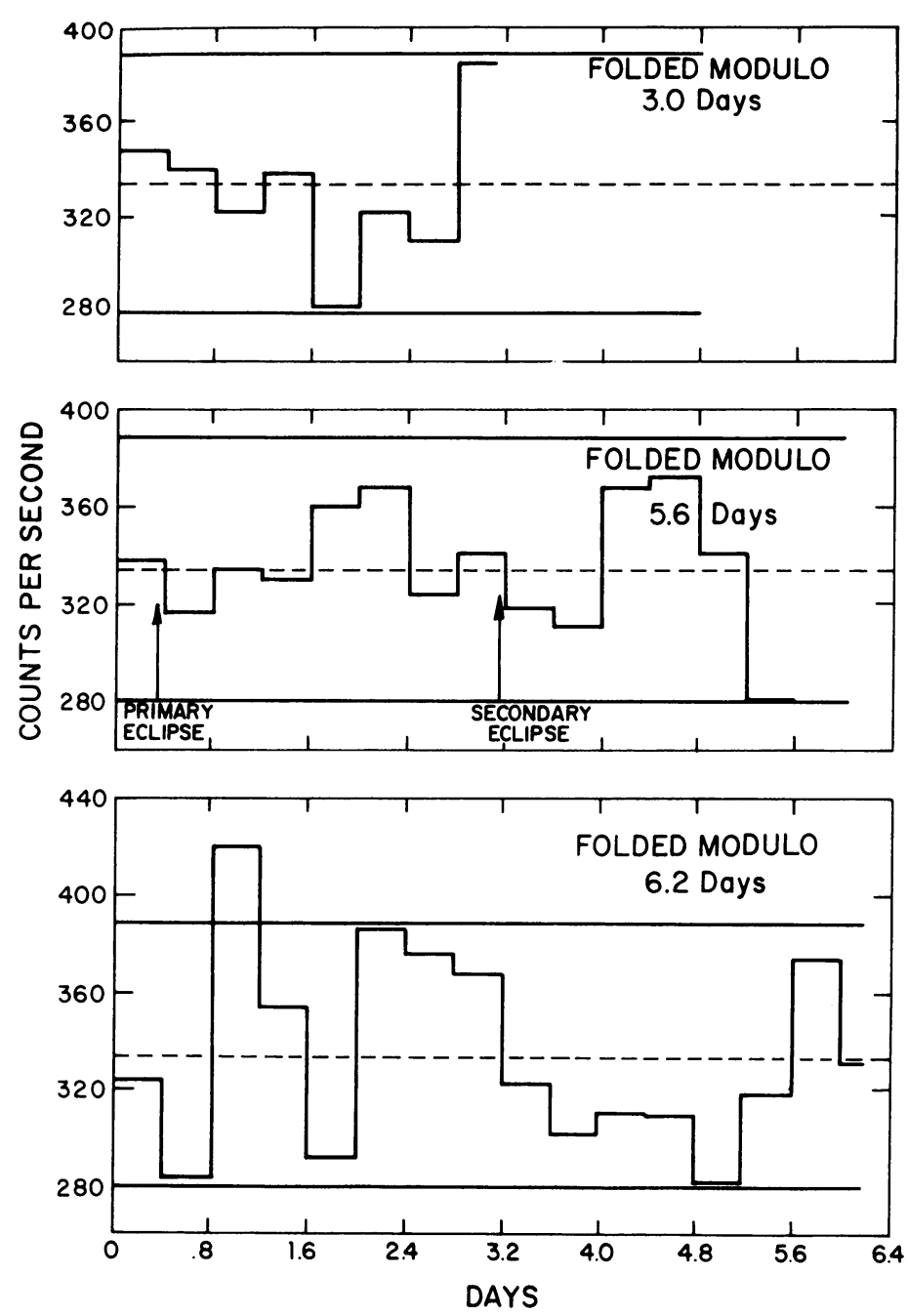

Fig. 3. Cygnus X-1 folded intensity, 17 December 1971 to 21 January $1972 ; 35$ days of $2-6 \mathrm{keV}$ data are shown folded modulo 3.0, 5.6, and 6.2 days. The dotted lines give the average intensity, and the solid lines are 2- $\sigma$ error bars.

$10-20 \mathrm{keV}$ flux increased by a factor of 2 . The figure also shows that at the same time the X-ray intensity changed, a weak radio source appeared at the Cyg X-1 location and was detected by the Westerbork and NRAO groups. Hjellming (1973) has recently reported analysis of additional radio data which shows the radio source first appeared some time between March 22 and March 31, essentially the time during which the $2-6 \mathrm{keV}$ X-ray intensity first headed downward. This correlated X-ray-radio behavior is the major evidence in addition to the positional data that Cyg X-1 is in fact identified with the optical and radio object.

With respect to the arguments concerning the mass of the secondary and thereby 

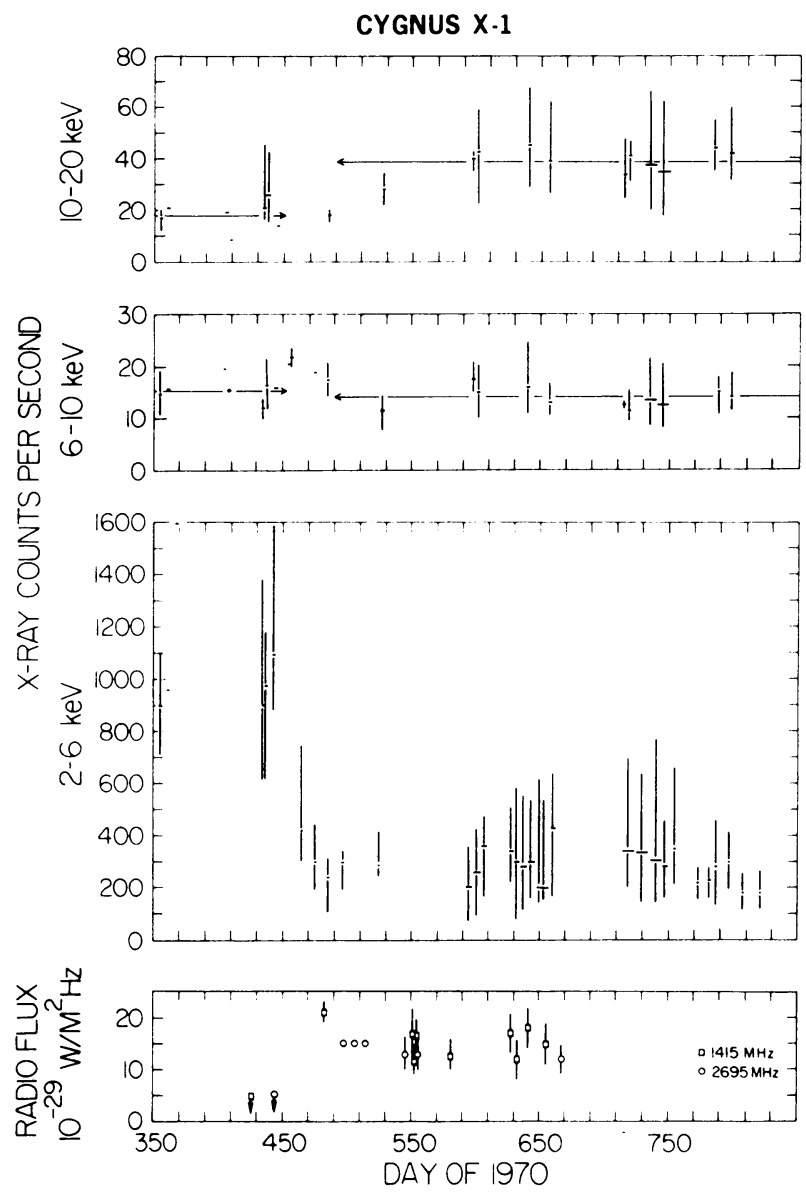

Fig. 4. 16 months of observations of Cygnus X-1. X-ray data are shown for three energy bands, 2-6 keV, 6-10 keV, and 10-20 keV, plotted vs. day of 1970. The transition discussed in the text occurred near day 450. The radio data are shown at the bottom of the figure, to which should be added the additional positive sighting on day 455 reported by Hjellming (1973).

the possibility of Cygnus X-1's being a black hole, there are three recent papers that approach the question. One paper by Bolton (1972b) uses the absorption line velocities, the HeII $4686 \AA$ emission line velocities, the $2.2 \mathrm{kpc}$ distance derived from the observed reddening and the equivalent width and velocity of the interstellar lines to determine values of $20 M_{\odot}$ for the primary, $13 M_{\odot}$ for the secondary, and an inclination angle of $26^{\circ}$. Cherepashchuk et al. (1973) use the absorption line velocities, the He II emission line velocities with some allowance that the emission region may not belong to the X-ray star, but may lie between the two stars (the possible flaw in Bolton's work), and the photoelectric observations showing 0.07 mag changes due to a tidally distorted system. Then taking into account limb darkening and gravity darkening, and making the not necessarily correct assumption that the primary fills its Roche lobe, they determine a primary mass between 10.7 and $22 M_{\odot}$, a secondary 
mass between 7.8 and $17 M_{\odot}$, and a distance of order $5 \mathrm{kpc}$ - although they appear to have neglected interstellar absorption effects and could therefore have overestimated the distance by a factor of 2. H. Mauder (1973) uses the mass function determined from the absorption line velocities, the possible distances allowed by the observed reddening and the absence of a bright infrared source which would be produced by an absorbing circumstellar shell around the source, the absence of any substantial reflection effects as demonstrated by the photoelectric observations and the X-ray to visible light energy ratio, and the photoelectric observations plus the assumption that the star cannot be any larger than its Roche lobe. He determines a self-consistent set of parameters that gives for a distance of $2 \mathrm{kpc}$, a primary mass of $25 M_{\odot}$ and a secondary mass between 6.0 and $7.3 M_{\odot}$.

What is the point of this rather lengthy, yet sketchy description? Since we are talking about the first possible observation of a black hole, we want to consider all the possibilities. The first optical papers suggested a mass of $\sim 20 M_{\odot}$ for the primary star based on a B0 Ib spectral typing. Among others, Trimble et al. (1973), and Faulkner (1973) pointed out that a helium burning star of much less mass could give a temperature and effective surface gravity identical to that observed. Since such a source would be at a distance of $1 \mathrm{kpc}$ at most, a knowledge of the distance is crucial for deciding the masses of the stars. Now the recent distance measurements that have been made by Bregman et al. (1973) and Margon et al. (1973) rule out the more exotic helium stars, determine that the primary is a massive supergiant, and thereby yield masses from 6 to $17 M_{\odot}$ for the secondary.

There are three links in the chain of arguments leading to our conclusion about Cygnus X-1 - (1) the identification, where location agreement plus the X-ray-radio correlation suggest the HDE 226868 secondary is the X-ray source; (2) the compactness, where the short time variations, accretion as an energy source, and the absence of substantial visible light emission from the secondary indicate that this object is compact; and (3) the mass estimates of $\geqslant 6 M_{\odot}$, which therefore indicate that Cygnus $\mathrm{X}-1$ is a black hole.

In the way of future work on this source, we have the promise of a 1 arcsec X-ray location from the HEAO-B X-ray telescope in the late 1970's to make the identification absolute, the large-area NRL experiment planned for the HEAO-A mission to study the intensity variations on very short time scales to pursue the compactness, and the continuing optical efforts to confirm the distance measurements.

\section{Cygnus $\mathrm{X}-3$}

If we now turn to Cygnus X-3, we find a different looking X-ray picture. Figure 5 shows the 2-6 keV Uhuru observation of Cygnus X-3 for 9 days in May 1972. The error bars contain both the statistical and systematic uncertainties. The data show intensity variations of about a factor of two with a period of $4.8 \mathrm{~h}$ first reported by Parsignault et al. (1972). Figure 6 shows the 9 days' data in three different energy bands from 1.8 to $10.0 \mathrm{keV}$ plotted folded with the 4.8 -h period. We see that the 

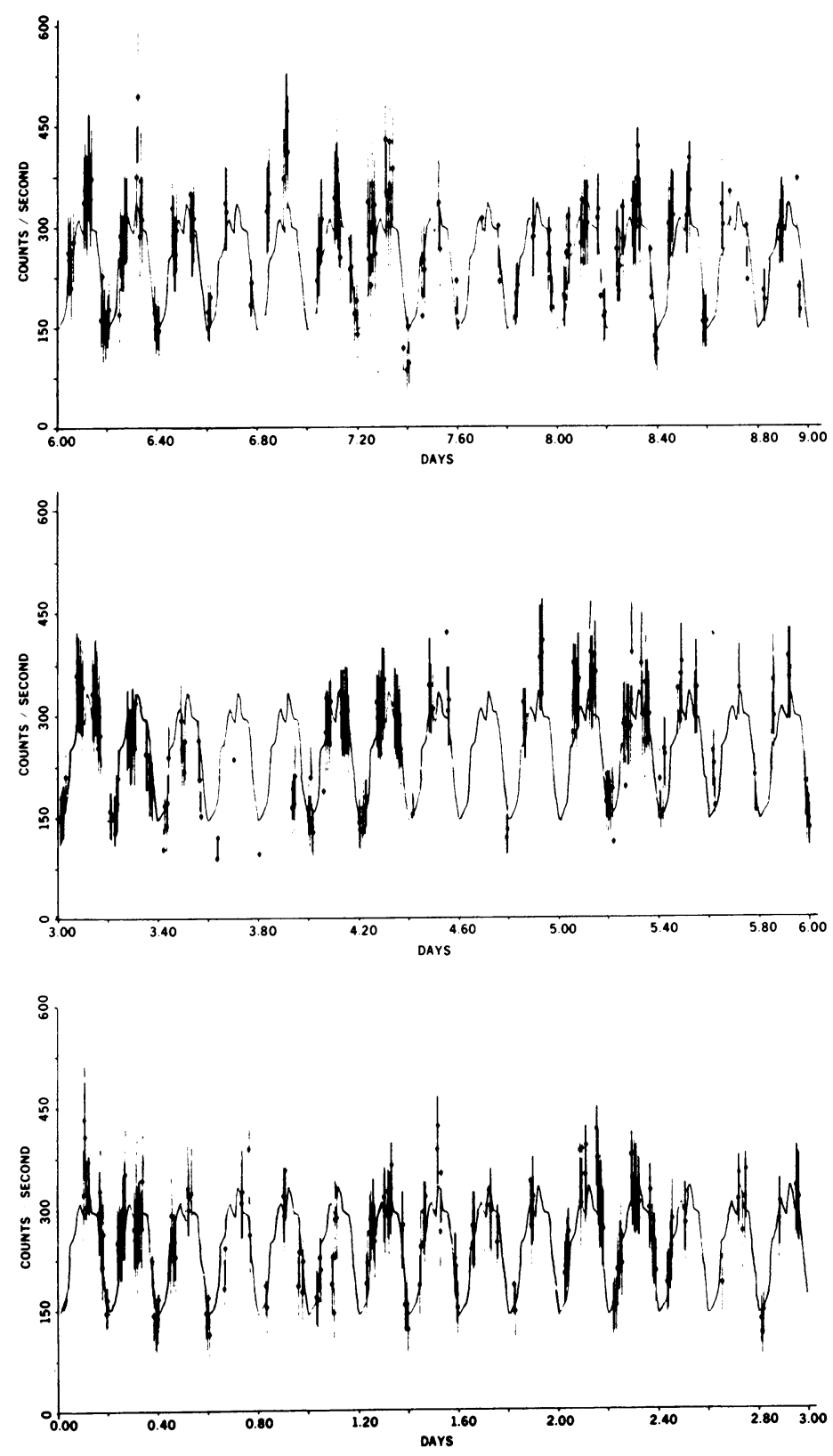

Fig. 5. Observations of Cygnus X-3 from 8 May to 17 May are plotted as a function of time. The $2-6 \mathrm{keV}$ intensities are given in counts $\mathrm{s}^{-1}$ and have been corrected for aspect; error bars include both statistical error and systematic error due to aspect correction. Also shown is the average light curve obtained by folding all the data modulo $4.80 \mathrm{~h}$ and finding the average intensity every $0.24 \mathrm{~h}$. 
CYGNUS $X \cdot 3$

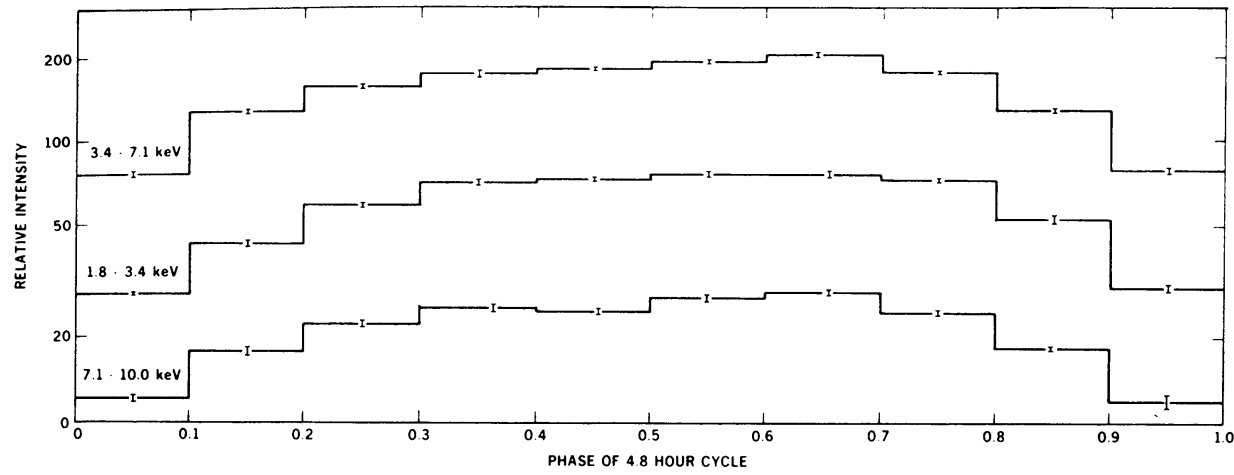

Fig. 6. Counting rate data for Cygnus X-3 for the same 9 days in May 1972 folded modulo $4.8 \mathrm{~h}$ for three energy bands. The intensity scale is logarithmic, and the data show that the $4.8-\mathrm{h}$ intensity variations are essentially energy independent.

minimum is observed in all three energy bands and that the intensity variations across the 4.8-h cycle are essentially independent of energy. Thus the minimum is not due to photoelectric absorption, which would show a strong energy dependence $\left(e^{-}(E a / E)^{8 / 3}\right)$. Among the possible models still being considered for this 4.8-h light curve are a binary system with a 4.8 -h orbital period and intensity variations caused either by geometrical obstruction or by different optical depths in different viewing directions due to only weakly energy dependent Compton scattering in a hot ionized cloud, an idea suggested by Gursky (1973). Compton scattering calculations by Tucker (1973) suggest an emitting region that would be of order $10^{8} \mathrm{~cm}$, a size consistent with short time scale fluctuations now observed on at least some occasions, indicating a compact component of the source. Other possibilities for the 4.8-h cycle would be pulsation or rotation of a single star.

For the 4.8-h cycle we have a new, tentative period of $0.1996515 \pm 0.0000032$ days constant from December 1970 through June 1971 and a period of $0.1996787 \pm 0.0000012$ from June 1971 to May 1972, an $8 \sigma$ statistical difference. A word of caution concerning this 1 part in $10^{4}$ increase in the period is in order. The method used to determine the phase of the minimum consists of fitting a sine function to the intensity variations. The period is then determined by dividing the time between minimum phases by the appropriate integer number of periods. Canizares et al. (1973) have reported evidence for changes in the shape of the light curve and we must investigate the effects of such a change on our analysis technique before considering the period change as definite. Changes in this period could prove very important in choosing among various models for the 4.8-h cycle.

Figure 7 shows several points over a 1.5 -yr period where we have determined the average intensity of Cygnus X-3. The points with the smallest error bars are data for which the average intensity was determined by the sine fitting technique; other points are obtained as daily averages of randomly selected points or as averages of selected 
CYG X-3: AVERAGE INTENSITY

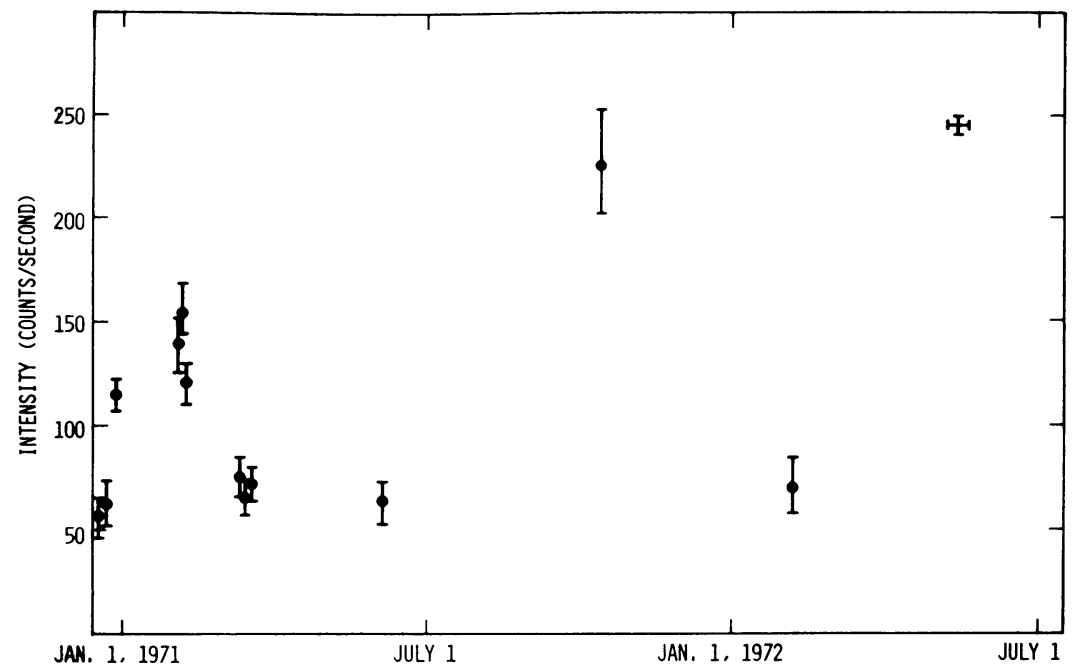

Fig. 7. Average 2-6 keV intensity for Cygnus X-3 on various days from January 1971 until July 1972. Data are corrected for aspect and the concept of 'average' is discussed in the text.

quick look data points at various phases of the 4.8-h cycle. The data suggest that Cyg X-3 may have average intensity levels which persist for times of months with transitions between levels sometimes observed. Average intensity levels of 60, 125 and 230 cts $\mathrm{s}^{-1}$ are seen at various times. This picture is somewhat similar to that observed for Cygnus X-1 and suggests that the radio data be checked against the X-ray observations, if possible, for possible long term correlated changes in average intensity.

Figure 8 shows new results for the X-ray intensity for 6 days at the time of the first September 1972 radio flare. We now include production data whereas our earlier report of no significant X-ray changes was based on quick look data only. We see that there are several points of high intensity on September 1, the day before the radio flare was first reported, although the radio data allow for the possibility of an earlier start up since the flare was first observed already in progress. The presence of X-ray intensities of at least $600 \mathrm{cts} \mathrm{s}^{-1}$, an intensity greater than ever previously observed for Cygnus X-3, and at least a factor of 2 higher than any intensities measured on August 30 and 31 indicates a connection between the $\mathrm{X}$-ray and radio behavior. This is also supported by preliminary observations of higher temperature energy spectra for the high intensity data points. This observation is important since it confirms the X-ray radio identification previously only suggested by positional coincidence of a few arcminutes. This identification is also confirmed as reported by Professor Boyd at the IAU General Assembly in Sydney by the very recent observation by Becklin et al. (1973) of a 4.8-h period in the infrared object identified with the Cyg X-3 radio source, with the infrared minimum in phase with the X-ray minimum.

Wallace Tucker and I have performed some very rough, back of the envelope cal- 
culations to see what the parameters of a system might be in which $10^{38} \mathrm{erg} \mathrm{s}^{-1}$ is produced in a synchrotron process $\mathrm{X}$-ray flare and $10^{33} \mathrm{erg} \mathrm{s}^{-1}$ is produced in a radio flare. We basically have a model in which the electrons are injected and first produce $\mathrm{X}$-rays in a small region with an intense magnetic field and then expand into a larger region with smaller $B$ and produce the radio emission. We assume an X-ray emitting region of order $10^{9} \mathrm{~cm}$ and a radio emitting region of order $10^{15} \mathrm{~cm}$.

Assuming a radio lifetime of 10 days we find as already determined by Gregory et al. (1972) a magnetic field of $\lesssim 5 \mathrm{G}$ in the radio emitting region; we now can determine a magnetic field of $5 \times 10^{9} \mathrm{G}$ in the $\mathrm{X}$-ray region, electron energies of $\sim 10$ $\mathrm{MeV}$, and number densities of $\sim 5 \times 10^{-2} \mathrm{~cm}^{-3}$ for the radio emitting region and $\sim 5 \times 10^{3} \mathrm{~cm}^{-3}$ for the $\mathrm{X}$-ray region.

\section{CYG X.3. LIGHT CURVE AROUND THE RADIO FLARE}

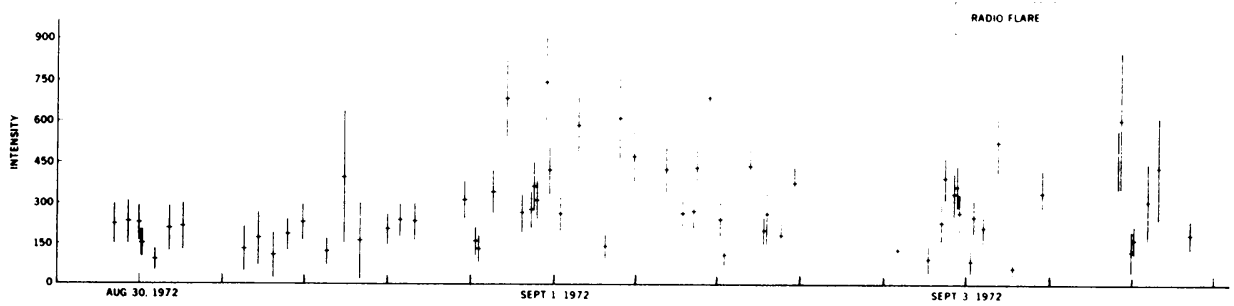

Fig. 8. 2-6 keV X-ray intensity for Cygnus X-3 in late August and early September 1972. The time of the first observation of the giant radio flare is indicated at 2200 on September 2 . Note the difference in the $\mathrm{X}$-ray behavior around September 1 compared to the preceding days.

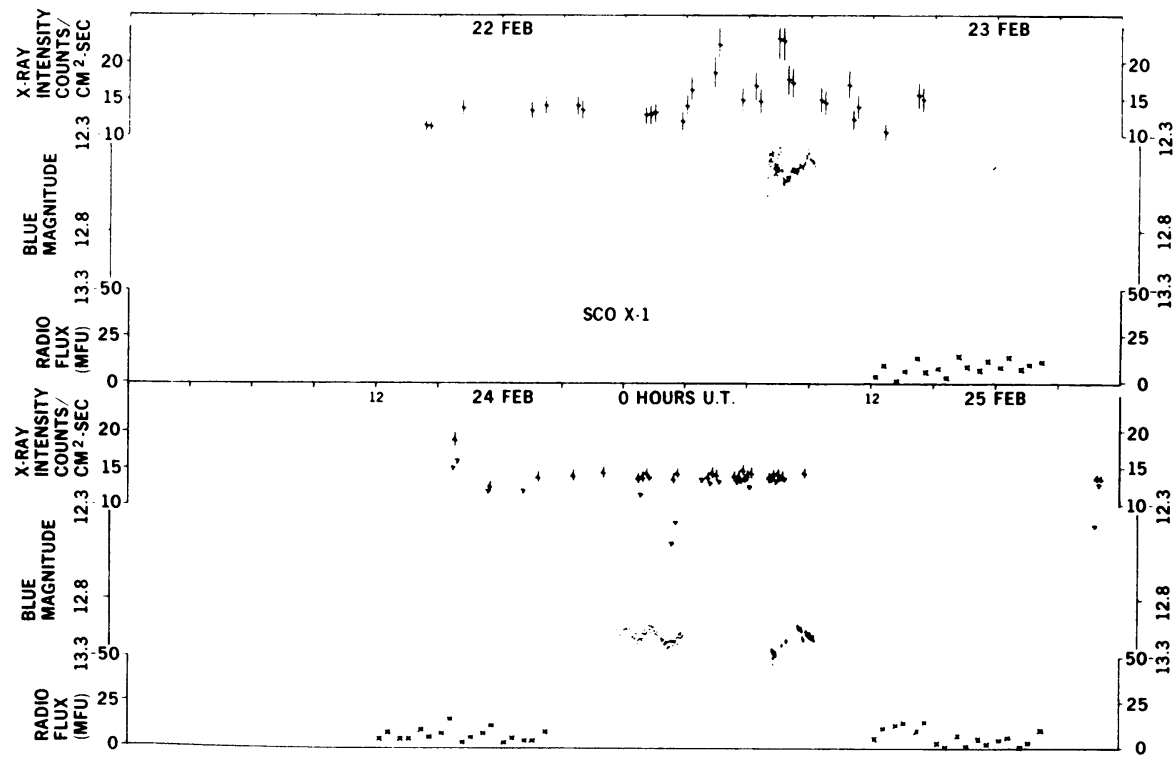

Fig. 9. Simultaneous X-ray, optical, and radio observations for Sco X-1 on 22-25 February 1971. The data are discussed in the text. 


\section{Scorpius X-1}

Turning to Sco X-1 we have considerably more detailed observations, particularly those obtained by collaborative efforts of a number of radio and optical observers together with Uhuru in February and March 1971.

Figure 9 shows the X-ray intensity in counts $\mathrm{cm}^{-2} \mathrm{~s}^{-1}$, the blue magnitude, and the radio intensity observed at NRAO and Westerbork in mJy.

The radio source is relatively quiet and weak on February 23, 24 and 25; the optical intensity is relatively bright and variable on February 23 and the X-ray source is also highly variable. On February 24 and 25 the blue-magnitude is fainter than $13 \mathrm{mag}$ and the X-ray emission is relatively low and quiet.

Figure 10 shows a continuation of this optical and X-ray behavior on February 26, with an enormous radio flare beginning around $18 \mathrm{~h}$ on February 26 . Unfortunately, the X-ray and optical observations are notably absent on the 2nd half of February 26 and on February 27, except for three hours of relatively faint blue observations. When the radio data resume at $12 \mathrm{~h}$ on February 27 , the flare has totally subsided, and then the X-ray and optical data for February 28 show low intensity and not much variability. Data such as these have been used to infer that the radio behavior is not related to the optical and X-ray emission, but the scarcity of X-ray and optical observations during much of the time on February 26 and 27 makes this conclusion unwarranted.

In Figure 11 we pick up Sco X-1 on March 23, 1971. Much of the data for March 23 and 24 show the blue magnitude brighter than $12.6 \mathrm{mag}$ and the $X$-ray source intense and varying (although there are some quiet X-ray times). When the data are

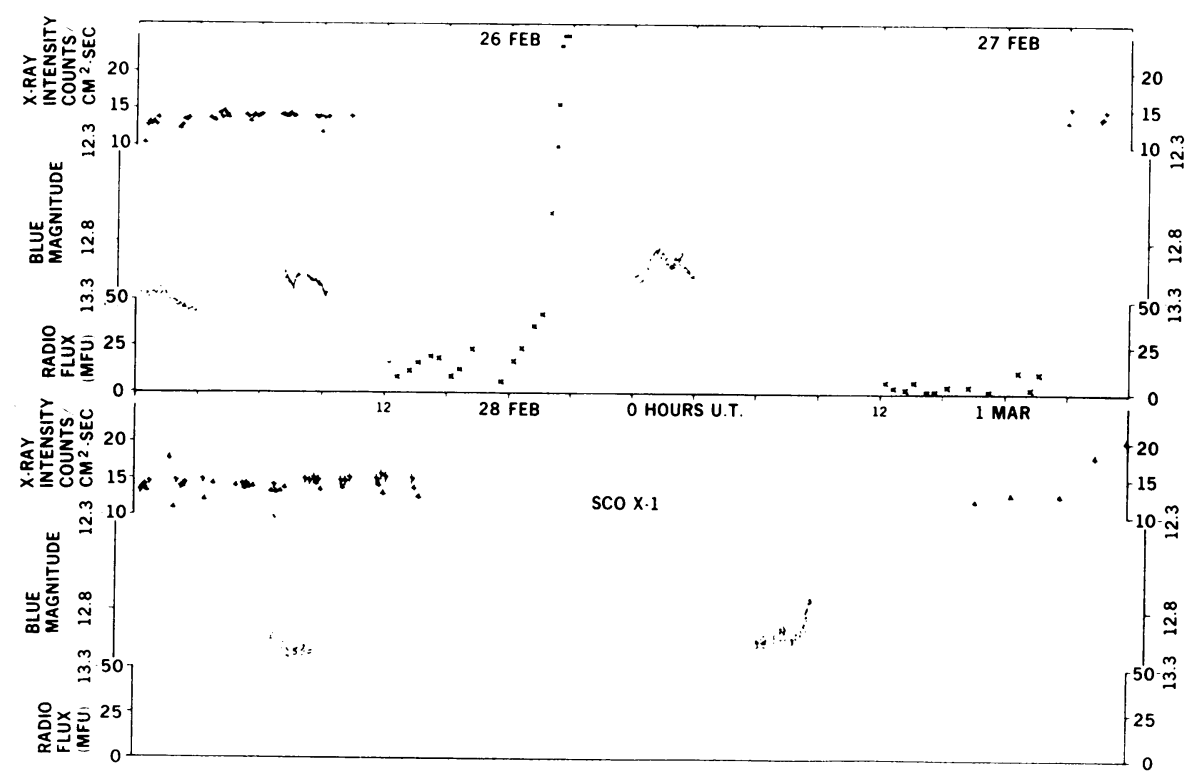

Fig. 10. Same as Figure 9 except for 26 February-1 March 1971. 


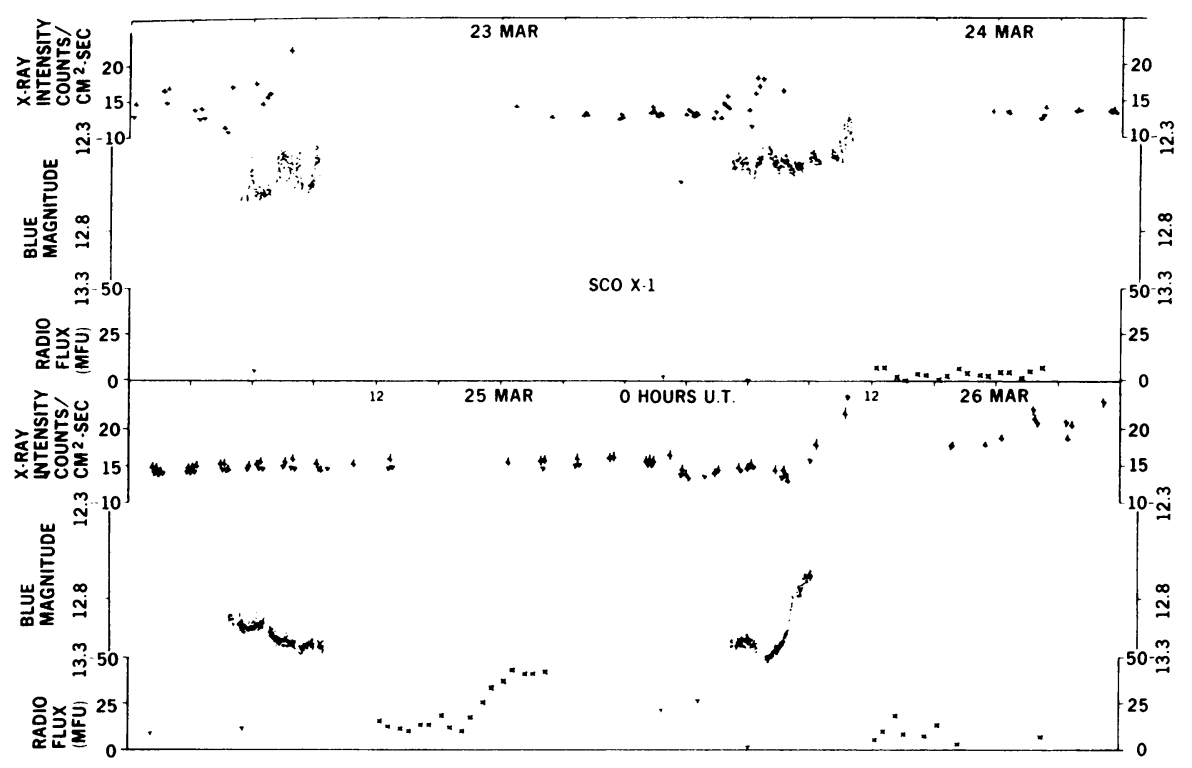

Fig. 11. Same as Figure 9 except for 23-26 March 1971.

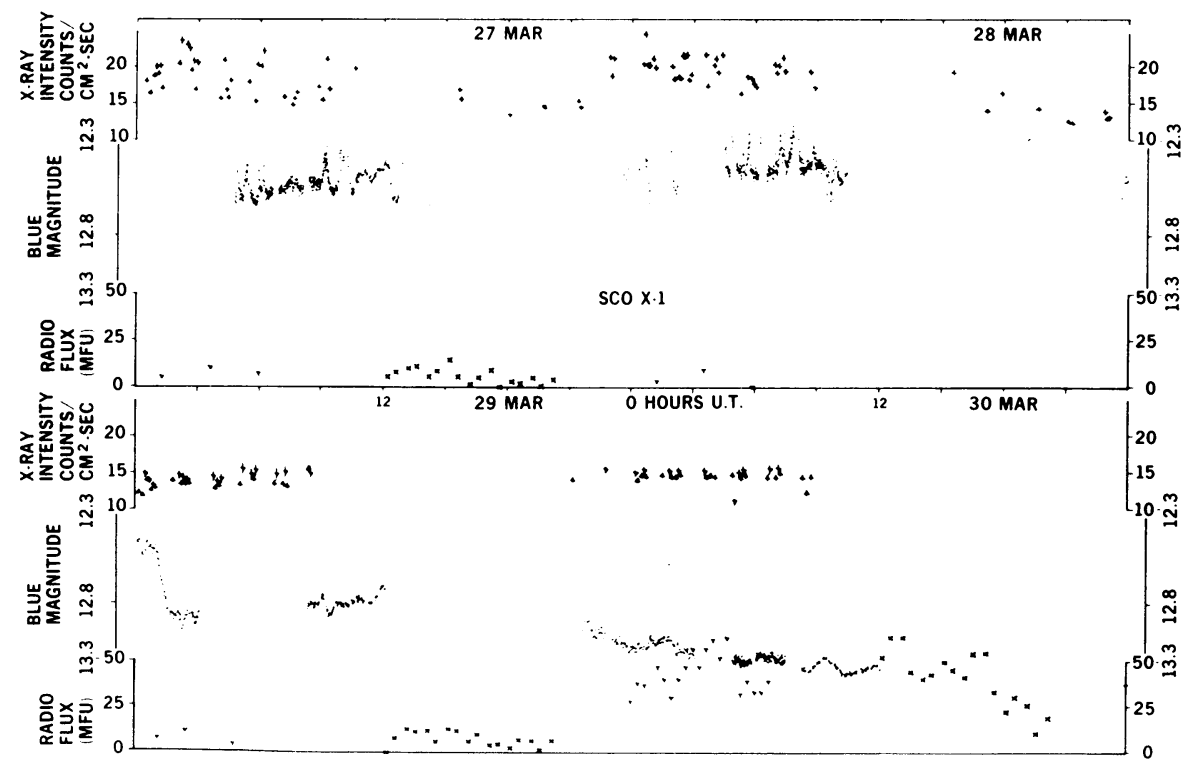

Fig. 12. Same as Figure 9 except for 27-30 March 1971. 
picked up on March 25, the X-ray intensity is very quiet and the optical is fainter than 13 mag. What would be helpful in attempting to understand how the source quieted down would have been more complete coverage on March 24 and 25.

Late on March 25, the radio data show a flare to about $500 \mathrm{mJy}$; the X-ray source remains low and quiet during this time, although there is a 6 hour gap in the data during the actual radio increase. On March 26 some $15 \mathrm{~h}$ after the radio flare, the optical brightness increases steeply, by several tenths of a magnitude in about an hour. About $1 \mathrm{~h}$ after the optical increase began, the X-ray intensity increases substantially and a bright, variable X-ray state follows. A few scattered radio points suggest that the radio flare may have disappeared some 2 to $4 \mathrm{~h}$ before the optical increase.

In Figure 12 we pick up the optical data showing the source still bright and highly variable on March 27 and 28 with a large decrease early on March 29. The X-ray points are also bright and variable on March 27 and most of March 28 and are definitely faint and relatively quiet on March 29. The March 28 data suggest that the $\mathrm{X}$-ray decrease may have preceded the optical decrease by as many as 6 to $8 \mathrm{~h}$. The $X$-ray and optical data remain low and quiet on March 29 and 30, with the radio data showing another flare, beginning between 21 and $24 \mathrm{~h}$ on March 29. The previous data from March 25 and 26 suggest that we either look for an optical and X-ray brightening some 15 hours after the start of the radio activity or some 2 to $4 \mathrm{~h}$ after the radio activity ends. Unfortunately, there are no X-ray data after $9 \mathrm{~h}$ on March

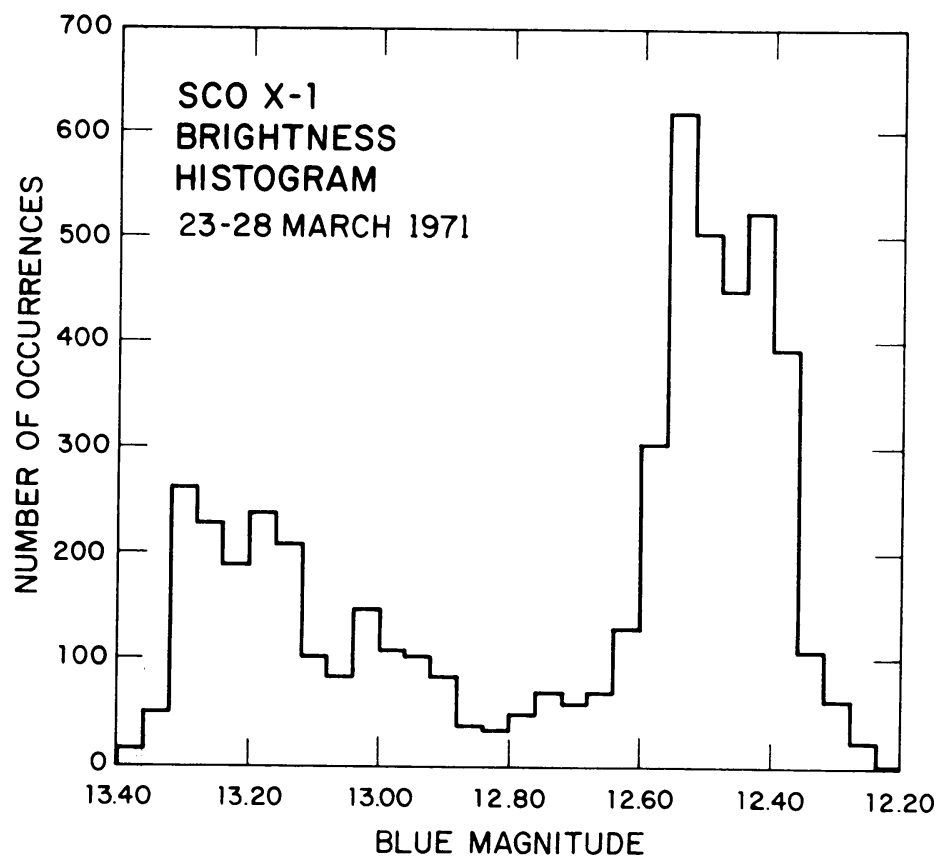

Fig. 13. Sco X-1 brightness histogram 23-28 March 1971. The number of occurrences of each blue magnitude is plotted vs. blue magnitude. The data indicate the clustering around $13.2 \mathrm{mag}$ and 12.5 mag as discussed in the text. 
30 nor any optical data at all on March 30, although optical data later on March 31 show the source faint. Thus, no conclusions can be drawn concerning repeated relationships between the radio and the optical and X-ray data.

Figure 13 shows a blue-magnitude occurrence histogram for March 23-28 suggesting two states for Sco X-1, with most of its time spent either bright or faint with little time in the middle. In Figure 14 we see the $X$-ray intensity versus blue magnitude for these days. This figure shows the correlation between the X-ray and optical. When the optical source is faint the $2-6 \mathrm{keV} \mathrm{X}$-ray intensity is low and varying only slightly. When the blue magnitude passes $12.6 \mathrm{mag}$, the X-ray intensity is greater by up to a factor of 2 and much more variable. This is also related to observations by Hiltner and Mook (1970) that Sco X-1 only flares in the optical when it is brighter than this same 12.6 mag. Figure 15 is 2 days of these same data where we show the X-ray temperature vs the $2-6 \mathrm{keV}$ intensity and find the source cooler when it is weaker and hotter and more variable when it is more intense. A better approach would have been

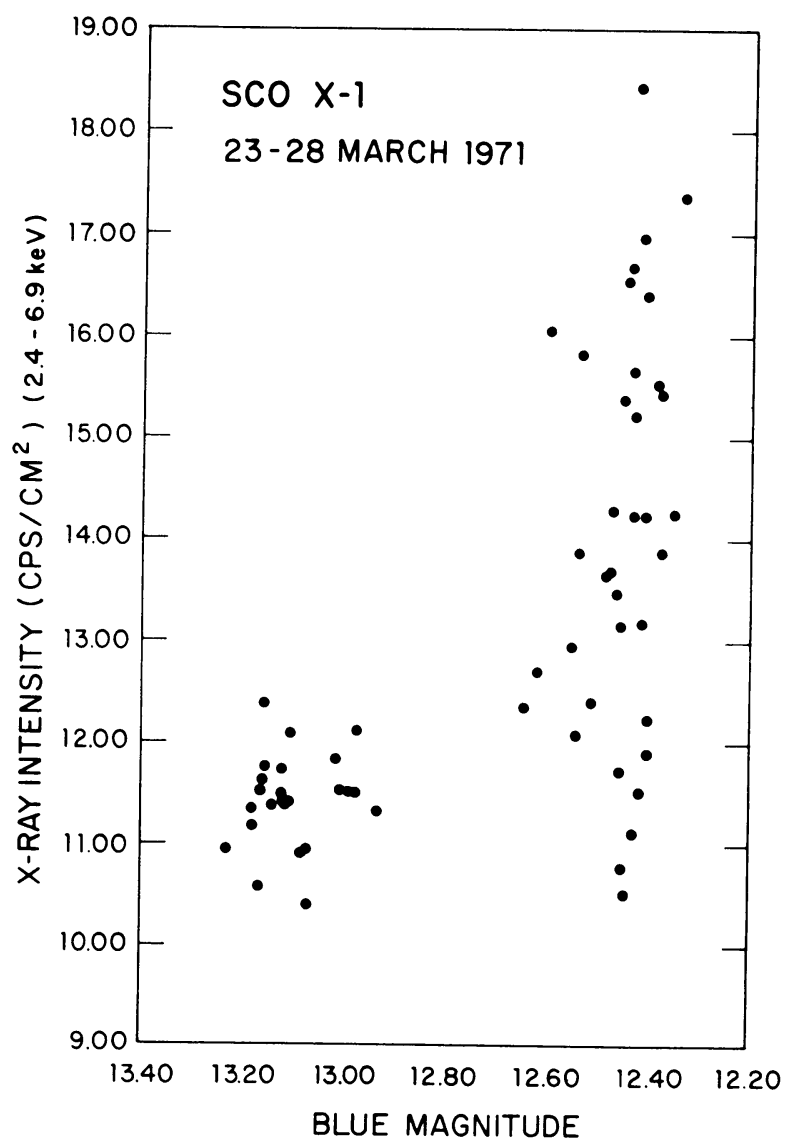

Fig. 14. Simultaneous X-ray-optical variability of Sco X-1. Each point represents a single, simultanous measurement of the X-ray and optical intensity of Sco X-1. Note the relatively quiet, faint state and the highly variable, bright state. 


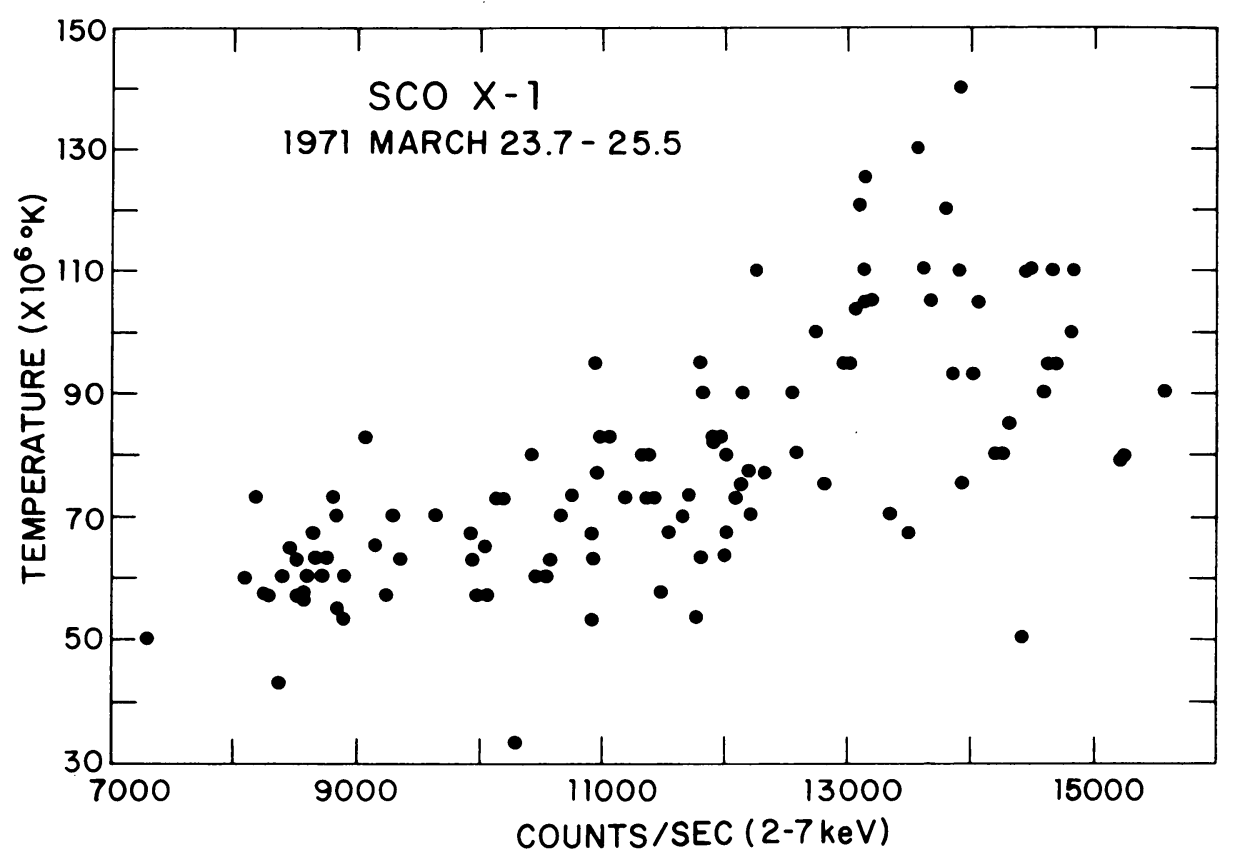

Fig. 15. Temperature versus X-ray intensity for Sco X-1. Each point represents a single temperature and $\mathrm{X}$-ray intensity observation. We see that as the source intensity increases the temperature increases and is more variable.

to plot temperature versus emission measure here, since the $2-6 \mathrm{keV}$ intensity is of course greatly affected by the exponential temperature factor.

An effort of this type was undertaken by Kitamura et al. (1971) with rocket data and optical observations which suggested a size of $10^{8}$ to $10^{9} \mathrm{~cm}$ and a density of $10^{15-16} \mathrm{~cm}^{-3}$ for the $\mathrm{X}$-ray emitting region. These conclusions were based on the observation that the emission measure decreased as the source became hotter. The Uhuru and MIT OSO 7 results suggest that this is not the case, but rather the emission measure increases as Sco X-1 becomes hotter. It may be that different laws govern this relationship depending on whether the source is in the bright flaring state or in the quiet, faint state. Present models suggest the radio emission occurs relatively far out from the X-ray emitting region, with the optical emission coming from a region also outside of but much closer to the $\mathrm{X}$-ray emitting region, resulting in the correlation between the optical and X-ray emission.

\section{Conclusion}

It should now be perfectly clear that not too much is perfectly clear. What is new is that at least some of the time the radio emission is more closely related to the X-ray emission than previously thought for these objects. Yet the time scales of a day or two that may be involved require future round-the-clock coordinated observations, 
such as suggested by Dr Hughes in Sydney for Cyg X-3 radio observations. There is also, no doubt, a wealth of information on the nature of the sources and the emission process still to be extracted in interpreting observations already made. One final interesting point recently made by Tucker (1973) is that the sources with intrinsic, variable low energy cut-offs - the eclipsing binaries - have not yet been observed as radio sources; this might be due to the high density gas near the source along our line of sight to the source with the high plasma frequency preventing the transmission of low frequency radio emission which may be present at the source. On the other hand, the X-ray data analyzed for Cyg X-1, Cyg X-3, and Sco X-1, as well as GX17+2 and $G X 9+1$, do not require intrinsic low energy absorption, which may mean the absence of the high density, radio absorbing gas near the source along the line of sight, and therefore the ability for us to observe radio emission. This suggests that the most viable candidates for future radio searches are those sources without large intrinsic cutoffs in their X-ray emission.

Note added. Further analysis has shown that the possible change in 4.8-h period for Cygnus X-3 cannot be isolated from effects due to changes in the shape of the X-ray light curve. Hence, we determine a period of $0.19967 \pm 0.00001$ days for all of the Uhuru observations of Cygnus X-3.

\section{Acknowledgements}

I gratefully acknowledge the contributions to this paper of several members of the Uhuru group at the HCO/SAO Center for Astrophysics and at AS\&E including Riccardo Giacconi, Ethan Schreier, Christine Jones, Bill Forman, Edwin Kellogg, Herb Gursky, Steven Murray, Dan Parsignault, Robert Leach and Wallace Tucker. I also appreciate the efforts of Hale Bradt and his co-workers in obtaining and analyzing the data from the coordinated observations of Sco X-1, and for allowing me to use the soon-to-be-published results.

\section{References}

Becklin, E. E., Hawkins, F. J., Mason, K. O., Matthews, K., Neugebauer, G., Sanford, P. W., and WynnWilliams, C. G.: 1973, preprint.

Bolton, C. T.: 1972a, Nature 235, 271.

Bolton, C. T.: 1972b, Nature Phys. Sci. 240, 124.

Braes, L. and Miley, G. K.: 1971, Nature 232, 246.

Bregman, J., Butler, D., Kemper, E., Koski, A., Kraft, R. P., and Stone, R. P. S.: 1973, preprint.

Canizares, C. R., McClintock, J. E., Clark, G. W., Lewin, W. H. G., Schnopper, H. W., and Sprott, G. F.: 1973, Nature Phys. Sci. 241, 28.

Cherepaschuk, A. M., Lyutiy, V. M., and Sunyaev, R. A.: 1973, Astron. Zh. 50, 3.

Faulkner, J.: 1973, Tucson Workshop on Compact X-Ray Sources.

Gregory, P. C., Kronberg, P. P., Seaquist, E. R., Hughes, V. A., Woodsworth, A., Viner, M. R., and Retallack, D.: 1972, Nature Phys. Sci. 239, 440.

Gursky, H.: 1973, private communication.

Hiltner, W. A. and Mook, D. W.: 1970, Ann. Rev. Astron. Astrophys. 8, 139.

Hjellming, R. M.: 1973, Astrophys. J. Letters 182, L29. 
Hjellming, R. M. and Wade, C. M.: 1971, Astrophys. J. Letters 168, L21.

Holt, S., Boldt, E., Schwartz, D., Serlemitsos, P., and Bleach, R.: 1971, Astrophys. J. Letters 166, L65.

Kitamura, T., Matsuoka, M., Miyamoto, S., Nakagawa, M., Oda, M., Ogawara, Y., Takagishi, K., Rao, U. R., Chitnis, E. V., Jayanthi, U. B., Prakasa-Rao, A. S., and Bhandari, S. M.: 1971, Astrophys. Space Sci. 12, 378.

Margon, B., Bowyer, S., and Stone, R.: 1973, Astrophys. J. Letters, to be published.

Mauder, H.: 1973, Astron. Astrophys., to be published.

Parsignault, D. R., Gursky, H., Kellogg, E. M., Matilsky, T., Murray, S., Schreier, E., Tananbaum, H., Giacconi, R., and Brinkman, A. C.: 1972, Nature 239, 123

Rappaport, S., Doxsey, R., and Zaumen, W.: 1971a, Astrophys. J. Letters 168, L43.

Rappaport, S., Zaumen, W., and Doxsey, R.: 1971b, Astrophys. J. Letters 168, L17.

Shulman, S., Fritz, G., Meekins, J., and Friedman, H.: 1971, Astrophys. J. Letters 168, 449.

Trimble, V., Rose, W. K., and Weber, J.: 1973, Monthly Notices Roy. Astron. Soc. 162, 1.

Tucker, W.: 1973, private communication.

Webster, B. L. and Murdin, P.: 1972, Nature 235, 37.

\section{Harvey Tananbaum}

Center for Astrophysics,

Harvard College Observatory/Smithsonian Institution,

60 Garden Street,

Cambridge, Mass. 02138, U.S.A.

\section{DISCUSSION}

Kaufmann: During the Cyg X-3 bursts in the first days of September 1972 we observed at Itapetinga (CRAAM), Brazil, very clear VLF propagation anomalies in the terrestrial lower ionosphere. Changes in diurnal phase variation were larger than $10 \%$. Since at nighttime in the ionospheric $E$-region the maximum electron production rate is somewhere in the $6-12 \mathrm{keV}$ range, the observed VLF anomalies would require $X$ ray emission in excess from Cyg X-3 or, even better, a steeper energy spectrum, with excesses at energies greater than $12 \mathrm{keV}$, as has been reported in this communication.

Lequeux: You showed variations in Cygnus X-3 in three different energy bands, and they look rather similar. Doesn't that contradict the statement that the low-energy cut-off in the source moves with the material?

Tananbaum: No, my statement was that the low energy cut-off does not vary across the 4.8-h cycle, precisely the point that rules out photo-electric absorption as a possible explanation of this 4.8 -h variation. 